\title{
On Friedrichs inequality, Helmholtz decomposition, vector potentials, and the div-curl lemma
}

Ben Schweizer 


\title{
On Friedrichs inequality, Helmholtz decomposition, vector potentials, and the div-curl lemma
}

\author{
Ben Schweizer ${ }^{1}$
}

September 23, 2016

\begin{abstract}
We study connections between four different types of results that are concerned with vector-valued functions $u: \Omega \rightarrow \mathbb{R}^{3}$ of class $L^{2}(\Omega)$ on a domain $\Omega \subset \mathbb{R}^{3}$ : Coercivity results in $H^{1}(\Omega)$ relying on div and curl, the Helmholtz decomposition, the construction of vector potentials, and the global div-curl lemma.
\end{abstract}

Key-words: Friedrichs inquality, coercivity, div-curl lemma, compensated compactness

\section{INTRODUCTION}

The original motivation of this text was to derive a variant of the div-curl lemma. This important lemma treats the convergence properties of products of two weakly convergent sequences of functions. Besides other applications, the lemma plays an important role in homogenization theory, in particular in nonperiodic homogenization problems. At some places, the name "compensated compactness" is used to refer to the div-curl lemma. We will use below the name "global" div-curl lemma to indicate that we are not satisfied with the distributional convergence of the product of functions, but that we want to obtain the convergence of the integral of the product.

The usual proof of the div-curl lemma is based on the construction of vector potentials, see e.g. [JKO94]. In the global div-curl lemma, the construction of potentials must be performed taking special care of appropriate boundary conditions. The proof of the div-curl lemma becomes shorter if it is based on a Helmholtz decomposition result. Once more, the global div-curl lemma requires a careful analysis of the boundary conditions.

Both, the construction of vector potentials and the proof of the Helmholtz decomposition, can be obtained from coercivity results involving divergence and curl of a function $u: \mathbb{R}^{3} \supset \Omega \rightarrow \mathbb{R}^{3}$. We have not been able to find a clear description of this connection in the literature. Moreover, the literature

\footnotetext{
${ }^{1}$ Technische Universität Dortmund, Fakultät für Mathematik, Vogelpothsweg 87, D-44227 Dortmund, Germany.
} 
discusses the coercivity results usually in a form that not strong enough to obtain the above-mentioned consequences.

In this text we present coercivity results in various forms and provide sketches of their proofs. We demonstrate how the other results can be obtained quite directly from the coercivity estimates. Moreover, we obtain these other results in a strong form, i.e. with a good control of boundary data. To summarize, we treat the following closely connected subjects and describe their most relevant connections:

(1) Coercivity results relying on div and curl

(2) The Helmholtz decomposition

(3) Construction of vector potentials

(4) The global div-curl lemma

More specifically, we will show the following: Let $\Omega$ be a domain for which the coercivity estimate of Item (1) holds. Then $\Omega$ permits statements as in Items (2)-(4) in a strong form.

Let us describe more clearly what is meant by the above items (1)-(4).

(1) The coercivity regards inequalities that allow to estimate, in the space $L^{2}(\Omega)$, all derivatives of a field $u: \Omega \rightarrow \mathbb{R}^{3}$ in terms of its divergence div $u$ : $\Omega \rightarrow \mathbb{R}$ and its rotation curl $u: \Omega \rightarrow \mathbb{R}^{3}$.

(2) In the Helmholtz decomposition we are interested in constructing, given $f \in L^{2}\left(\Omega, \mathbb{R}^{3}\right)$, two functions $\phi$ and $w$ such that $f=\nabla \phi+w$ with $\operatorname{div} w=0$. In strong Helmholtz decomposition results, we want to write curl $\psi$ instead of $w$ and impose boundary conditions on $\psi$.

(3) Construction of vector potentials: Given a field $f: \Omega \rightarrow \mathbb{R}^{3}$ with $\operatorname{div} f=$ 0 , we want to find a potential $\psi: \Omega \rightarrow \mathbb{R}^{3}$ such that $f=$ curl $\psi$, again imposing boundary conditions on $\psi$.

(4) In the div-curl lemma one considers sequences $f_{k} \rightarrow f$ and $p_{k} \rightarrow p$ as $k \rightarrow \infty$ in $L^{2}\left(\Omega, \mathbb{R}^{3}\right)$. The additional information is that both $\left\|\operatorname{div} f_{k}\right\|_{L^{2}(\Omega)}$ and $\|$ curl $p_{k} \|_{L^{2}(\Omega)}$ are bounded sequences. One is interested in the product $f_{k} \cdot p_{k}$. In the standard div-curl lemma, one obtains the distributional convergence $f_{k}$. $p_{k} \rightarrow f \cdot p$ as $k \rightarrow \infty$. We are interested in the global div-curl lemma, which provides $\int_{\Omega} f_{k} \cdot p_{k} \rightarrow \int_{\Omega} f \cdot p$ as $k \rightarrow \infty$.

We note that the coercivity result (1) requires quite strong assumptions on $\Omega \subset \mathbb{R}^{3}$ (the regularity $\mathcal{C}^{1,1}$ and simply connectedness or, alternatively, the convexity of $\Omega$ ). On the other hand, given (1), the results (2)-(4) can be derived easily in strong forms. In particular, we obtain these results with a control of the boundary data and with natural estimates.

Applications. As already mentioned, the div-curl lemma plays a crucial role in the derivation of homogenization limits, in particular if one follows the Russian approach, which is well adapted to perform stochastic homogenization limits, see [JKO94]. A recent application is the non-periodic homogenization of plasticity 
equations. For such nonlinear non-periodic problems, the so-called "needleproblem approach" was developed in [SV11]. The crucial step in this approach is to find, given a sequence of functions $u^{\varepsilon}$ on a domain $\Omega$, a triangulation of $\Omega$ such that the global div-curl lemma can be applied on every simplex of the triangulation. The method was applied to perform the homogenization of plasticity equation in [HS15, HS14].

An observation. Since our proofs are based on coercivity estimates, we start with an observation regarding divergence and curl of functions.

Remark 1.1. Let $\Omega \subset \mathbb{R}^{3}$ be a bounded domain. We consider functions $u$ : $\Omega \rightarrow \mathbb{R}^{3}$ with vanishing boundary values, $u \in H_{0}^{1}(\Omega)$. For such functions, the control of curl $u$ and $\operatorname{div} u$ in $L^{2}(\Omega)$ is equivalent to the control of the full gradient in $L^{2}(\Omega)$. Indeed, for $u \in H_{0}^{1}(\Omega)$ (i.e.: all components of $u$ vanish along the boundary), the following calculation is valid

$$
\begin{aligned}
\int_{\Omega}\left\{|\nabla \cdot u|^{2}+|\operatorname{curl} u|^{2}\right\} & =\int_{\Omega}[-\nabla(\nabla \cdot u)+\operatorname{curl} \operatorname{curl} u] \cdot u \\
& =\int_{\Omega}[-\Delta u] \cdot u=\int_{\Omega}|\nabla u|^{2} .
\end{aligned}
$$

Remark 1.1 indicates that all derivatives of $u$ are controlled by $\nabla \cdot u$ and curl $u$ - at least up to contributions from boundary integrals.

We note that Remark 1.1 has some similarity with the trivial Korn's inequality (Korn's inequality for functions with vanishing boundary values): The integral over squared gradients is (up to a factor 2) identical to the integral over squared symmetrized gradients. This is similar to equation (1.1). Korn's inequality (the non-trivial version) shows that, indeed, the full gradient of $u$ can be estimated in terms of the symmetrized gradient of $u$.

\section{Notation}

In the following, $\Omega \subset \mathbb{R}^{3}$ always denotes a bounded open set, further properties will be specified when needed. For Lipschitz domains $\Omega$, we denote the exterior normal by $\nu: \partial \Omega \rightarrow \mathbb{R}^{3}$ ( $\nu$ is defined almost everywhere on the boundary).

We use the space $H(\Omega$, curl $):=\left\{u \in L^{2}\left(\Omega, \mathbb{R}^{3}\right) \mid\right.$ curl $\left.u \in L^{2}\left(\Omega, \mathbb{R}^{3}\right)\right\}$, where curl $u$ is understood in the distributional sense. The norm on this space is $\|u\|_{L^{2}}+\|\operatorname{curl} u\|_{L^{2}}$. The subspace of functions with vanishing boundary condition is $H_{0}(\Omega$, curl $)=\left\{u \in H(\Omega\right.$, curl $\left.)|\nu \times u|_{\partial \Omega}=0\right\}$. We emphasize that, since only the curl of $u$ is controlled, only tangential boundary data can be evaluated in the sense of traces. Since trace estimates require Lipschitz boundaries, we define the space $H_{0}(\Omega$, curl $)$ with a weak formulation as follows:

$$
H_{0}(\Omega, \operatorname{curl}):=\left\{u \in H(\Omega, \operatorname{curl}) \mid \int_{\Omega} \operatorname{curl} u \cdot \eta=\int_{\Omega} u \cdot \operatorname{curl} \eta \forall \eta \in H^{1}\left(\Omega, \mathbb{R}^{3}\right)\right\} .
$$


Similarly, the space of functions with divergence in $L^{2}(\Omega)$ can be defined as: $H(\Omega, \operatorname{div}):=\left\{u \in L^{2}\left(\Omega, \mathbb{R}^{3}\right) \mid \operatorname{div} u \in L^{2}\left(\Omega, \mathbb{R}^{3}\right)\right\}$ and the corresponding space with vanishing boundary data is $H_{0}(\Omega, \operatorname{div})=\left\{u \in H(\Omega, \operatorname{div})|\nu \cdot u|_{\partial \Omega}=0\right\}$, defined as

$$
H_{0}(\Omega, \operatorname{div}):=\left\{u \in H(\Omega, \operatorname{div}) \mid \int_{\Omega}(\operatorname{div} u) \eta=-\int_{\Omega} u \cdot \nabla \eta \forall \eta \in H^{1}(\Omega, \mathbb{R})\right\} .
$$

We emphasize that the index 0 enforces in both cases that certain components of the vector field vanish on the boundary; these are tangential components in the case of $H_{0}(\Omega$, curl $)$ and normal components in the case of $H_{0}(\Omega$, div $)$.

Following [ABDG98], we use the space $X(\Omega):=H(\Omega$, curl $) \cap H(\Omega$, div $)$ and the two subspaces

$$
\begin{aligned}
& X_{N}(\Omega):=H_{0}(\Omega, \operatorname{curl}) \cap H(\Omega, \operatorname{div})=\left\{u \in X(\Omega)|\nu \times u|_{\partial \Omega}=0\right\}, \\
& X_{T}(\Omega):=H(\Omega, \operatorname{curl}) \cap H_{0}(\Omega, \operatorname{div})=\left\{u \in X(\Omega)|\nu \cdot u|_{\partial \Omega}=0\right\} .
\end{aligned}
$$

We note that the boundary values $\nu \times\left. u\right|_{\partial \Omega}$ are well defined in the sense of distributions for functions $u \in H\left(\Omega\right.$, curl). Similarly, $\left.\nu \cdot u\right|_{\partial \Omega}$ is well defined in the sense of distributions for functions $u \in H(\Omega$, div $)$.

\section{FriedRICHS INEQUALITY}

Many references are available for the following coercivity estimate. See e.g. (11) in [Lei68] or Corollary 2.2 in [Cos91], or Theorems 2.9 and 2.12 in [ABDG98].

We emphasize that the coercivity estimate of Theorem 3.1 remains valid on convex Lipschitz domains (the regularity $\partial \Omega \in \mathcal{C}^{1,1}$ is replaced by the convexity requirement), see Theorem 2.17 in [ABDG98].

Theorem 3.1 (Coercivity estimate). Let $\Omega$ be a bounded Lipschitz domain with $\partial \Omega \in \mathcal{C}^{1,1}$. Then there exists a coercivity constant $C_{C}>0$ such that

$$
\|u\|_{H^{1}}^{2} \leq C_{C} \int_{\Omega}\left\{|\nabla \cdot u|^{2}+|\operatorname{curl} u|^{2}+|u|^{2}\right\}
$$

for every $u \in X_{T}(\Omega)$. The constant $C_{C}$ can be chosen such that (3.1) holds also for every $u \in X_{N}(\Omega)$.

On the proof. The proof of (3.1) relies on the fact that for $u$ in either $X_{T}(\Omega)$ or $X_{N}(\Omega)$ critical boundary terms in the calculation (1.1) cancel. The remaining terms are products containing the curvature of the boundary and squares of values of $u$ on the boundary. It is important that, in the boundary integrals, no terms containing derivatives of $u$ remain. Moreover, for convex domains, the remaining terms have the good sign (see Lemma 2.11 in [ABDG98]). Combining the calculation (1.1) with a trace estimate for $u$ and an interpolation, one obtains (3.1). The full proof requires density results in the spaces $X_{T}(\Omega)$ and $X_{N}(\Omega)$. 
Our next step is to improve inequality (3.1) so that the $L^{2}(\Omega)$-norm of $u$ does not appear on the right hand side. We call the result a Friedrichs inequality.

Let us describe why we call the result a Friedrichs inequality: The above sketch of proof (more precisely, the positivity of boundary contributions for convex domains) suggests that the following inequality holds on convex domains with $C_{F}=1$ :

$$
\|\nabla u\|_{L^{2}\left(\Omega, \mathbb{R}^{3}\right)}^{2} \leq C_{F} \int_{\Omega}\left\{|\nabla \cdot u|^{2}+|\operatorname{curl} u|^{2}\right\}
$$

Inequality (3.2) is known as Friedrichs second inequality, see e.g. Theorem 3.1 in [Sar82]. Using a general constant $C_{F}$ in (3.2) is necessary for non-convex domains. We note that $(3.2)$ for $L^{p}(\Omega)$-spaces is treated in [vW92] with methods from potential theory. Regarding the result in space dimension 2 we refer to [KN84], Theorem 4.3.

We want to improve (3.2) and estimate the full $H^{1}$-norm.

Corollary 3.2 (Friedrichs inequality). Let $\Omega$ be a simply connected bounded Lipschitz domain. We assume that (3.1) holds (we recall that $\partial \Omega \in \mathcal{C}^{1,1}$ or convexity of $\Omega$ is sufficient). Then there exists a constant $C_{F}>0$ such that

$$
\|u\|_{H^{1}\left(\Omega, \mathbb{R}^{3}\right)}^{2} \leq C_{F} \int_{\Omega}\left\{|\nabla \cdot u|^{2}+|\operatorname{curl} u|^{2}\right\}
$$

holds for all functions $u$ in the space $X_{T}(\Omega)$. The same estimate holds for every $u \in X_{N}(\Omega)$.

Proof. We argue by contradiction. Let $\left(u_{k}\right)_{k}$ be a sequence with $\left\|u_{k}\right\|_{H^{1}(\Omega)}=1$ for every $k$ and with $\nabla \cdot u_{k} \rightarrow 0$ and curl $u_{k} \rightarrow 0$ in $L^{2}(\Omega)$. Rellich compactness allows to extract a subsequence and to find $u \in H^{1}(\Omega)$ such that $u_{k} \rightarrow u$ in $H^{1}(\Omega)$ and $u_{k} \rightarrow u$ in $L^{2}(\Omega)$. Weak limits coincide with distributional limits, hence $\nabla \cdot u=0$ and $\operatorname{curl} u=0$.

The curl-free function $u$ has a potential, $u=\nabla \Phi$ for some $\Phi \in H^{1}(\Omega)$. This fact is known as Poincaré lemma, we use at this point that $\Omega$ is simply connected. The potential can be constructed with line integrals; the extension to functions $u \in L^{2}(\Omega)$ is straightforward using density of smooth functions. The potential $\Phi$ solves $\Delta \Phi=0$ because of $\nabla \cdot u=0$. Furthermore, the boundary condition $u \in X_{T}(\Omega)$ implies that $\Phi$ has a vanishing normal derivative on $\partial \Omega$. The boundary condition $u \in X_{N}(\Omega)$ implies that tangential components of $\nabla \Phi$ vanish on the boundary, hence $\Phi$ can be chosen in $H_{0}^{1}(\Omega)$. In both cases, due to $\Delta \Phi=0$, the potential $\Phi$ is a constant function and $u$ vanishes.

The fact $u_{k} \rightarrow u=0$ in $L^{2}(\Omega)$ implies that the three terms on the right hand side of (3.1) vanish in the limit $k \rightarrow \infty$ for the sequence $u_{k}$. Inequality (3.1) yields $\left\|u_{k}\right\|_{H^{1}} \rightarrow 0$, which is the desired contradiction. 


\section{Helmholtz DeCOMPosition}

We formulate a strong Helmholtz decomposition result in Theorem 4.2. In order to explain why we call Theorem 4.2 a strong Helmholtz decomposition result, let us first state and prove an elementary version.

Proposition 4.1 (Elementary Helmholtz decomposition). Let $\Omega \subset \mathbb{R}^{3}$ be $a$ bounded domain. Then there exists a constant $C_{H}>0$ such that, for every vector field $f \in L^{2}\left(\Omega, \mathbb{R}^{3}\right)$, the following holds:

(1) Imposing a boundary condition for $w$. There exist $\phi: \Omega \rightarrow \mathbb{R}$ and $w: \Omega \rightarrow \mathbb{R}^{3}$ such that

$$
\begin{aligned}
& f=\nabla \phi+w, \quad \phi \in H^{1}(\Omega, \mathbb{R}), \\
& w \in W_{0}:=\left\{w \in L^{2}(\Omega) \mid \int_{\Omega} w \cdot \nabla \varphi=0 \forall \varphi \in H^{1}(\Omega)\right\} .
\end{aligned}
$$

(2) Imposing a boundary condition for $\phi$. There exist $\phi: \Omega \rightarrow \mathbb{R}$ and $w: \Omega \rightarrow \mathbb{R}^{3}$ such that

$$
\begin{aligned}
& f=\nabla \phi+w, \quad \phi \in H_{0}^{1}(\Omega, \mathbb{R}), \\
& w \in W:=\left\{w \in L^{2}(\Omega) \mid \int_{\Omega} w \cdot \nabla \varphi=0 \forall \varphi \in H_{0}^{1}(\Omega)\right\} .
\end{aligned}
$$

Both decompositions are valid with the estimate

$$
\|\phi\|_{H^{1}(\Omega)}+\|w\|_{L^{2}(\Omega)} \leq C_{H}\|f\|_{L^{2}(\Omega)} .
$$

Proof. For Item (1), we define $\phi \in H^{1}(\Omega)$ as the solution of the Neumann problem

$$
\int_{\Omega} \nabla \phi \cdot \nabla \varphi=\int_{\Omega} f \cdot \nabla \varphi \quad \forall \varphi \in H^{1}(\Omega) .
$$

The solution exists by the Lax-Milgram theorem in the space of $H^{1}$-functions with vanishing mean value. With this choice of $\phi$, the remainder $w:=f-\nabla \phi$ satisfies $w \in W_{0}$ by definition.

For Item (2), we define $\phi \in H_{0}^{1}(\Omega)$ as the solution of the Dirichlet problem

$$
\int_{\Omega} \nabla \phi \cdot \nabla \varphi=\int_{\Omega} f \cdot \nabla \varphi \quad \forall \varphi \in H_{0}^{1}(\Omega) .
$$

The solution exists by the Lax-Milgram theorem in $H_{0}^{1}(\Omega)$. With this choice of $\phi$, the remainder $w:=f-\nabla \phi$ satisfies $w \in W$ by definition.

In both cases, due to the solution estimate of the Lax-Milgram theorem, the norm of $\phi$ in $H^{1}(\Omega)$ and, hence, the norm of $w$ in $L^{2}(\Omega)$ are controlled by the norm of $f$ in $L^{2}(\Omega)$.

We next show a stronger Helmholtz decomposition result. Here, we write the solennoidal function $w$ as the curl of a vector potential $\psi$. Furthermore, we can 
prescribe a boundary condition for the vector potential. Again, all norms are controlled by the datum $f$.

Theorem 4.2 (Helmholtz decomposition with vector potential). Let $\Omega \subset \mathbb{R}^{3}$ be a simply connected bounded Lipschitz domain with boundary $\partial \Omega$ of class $\mathcal{C}^{1,1}$. Then there exists a constant $C_{H}>0$ such that, for every vector field $f \in L^{2}\left(\Omega, \mathbb{R}^{3}\right)$, we have:

(1) Imposing a boundary condition for $\psi$. There exist $\phi: \Omega \rightarrow \mathbb{R}$ and $\psi: \Omega \rightarrow \mathbb{R}^{3}$ such that

$$
f=\nabla \phi+\operatorname{curl} \psi, \quad \phi \in H^{1}(\Omega, \mathbb{R}), \quad \nabla \cdot \psi=0, \quad \psi \in X_{N}(\Omega) .
$$

(2) Imposing a boundary condition for $\phi$. There exist $\phi: \Omega \rightarrow \mathbb{R}$ and $\psi: \Omega \rightarrow \mathbb{R}^{3}$ such that

$$
f=\nabla \phi+\operatorname{curl} \psi, \quad \phi \in H_{0}^{1}(\Omega, \mathbb{R}), \quad \nabla \cdot \psi=0, \quad \psi \in X_{T}(\Omega) .
$$

In both cases, the decomposition satisfies the estimate

$$
\|\phi\|_{H^{1}(\Omega)}+\|\psi\|_{H^{1}(\Omega)} \leq C_{H}\|f\|_{L^{2}(\Omega)} .
$$

Remark 4.3. Many parts of Theorem 4.2 remain valid under the following weaker assumption $\left(\mathrm{A}_{1}\right)$ on $\Omega$ :

$\left(\mathrm{A}_{1}\right)$ Let $\Omega$ be a bounded Lipschitz domain such that the Friedrichs inequality (3.3) holds.

Item (1) of the Theorem remains valid without any changes in the proof. Instead, our proof of Item (2) makes use of the $\mathcal{C}^{1,1}$-regularity of the boundary.

In order to clearify the connection with Proposition 4.1, we note the following: With $\psi$ as in Item (1) above, there holds $w:=$ curl $\psi \in W_{0}$ (as in Item (1) of Proposition 4.1). Indeed, for $\varphi \in H^{1}(\Omega)$,

$$
\int_{\Omega} w \cdot \nabla \varphi=\int_{\Omega} \operatorname{curl} \psi \cdot \nabla \varphi=\int_{\Omega} \psi \cdot \operatorname{curl} \nabla \varphi=0 .
$$

\section{Proof. Proof of Item (1).}

Step 1. Construction of $\phi$. In this first step, we compensate the divergence $\nabla \cdot f$ and the normal boundary data $f \cdot \nu$ with a scalar potential $\phi$ (as in (4.6) in the proof of Item (1) of Proposition 4.1). We define $\phi \in H^{1}(\Omega, \mathbb{R})$ as the solution with vanishing average of the Neumann problem

$$
\int_{\Omega} \nabla \phi \cdot \nabla \varphi=\int_{\Omega} f \cdot \nabla \varphi \quad \forall \varphi \in H^{1}(\Omega, \mathbb{R}) .
$$

The solution $\phi$ satisfies the estimate (4.10). In the rest of the proof our aim is to write the function $\tilde{f}:=f-\nabla \phi \in W_{0}$ as the curl of a vector potential.

Step 2. Construction of $\psi$. We introduce the bilinear form

$$
b(u, v):=\int_{\Omega}\{(\nabla \cdot u)(\nabla \cdot v)+(\operatorname{curl} u) \cdot(\operatorname{curl} v)\}
$$


on the space $X_{N}(\Omega)$ of (2.3). We consider the following auxiliary problem: Find $\psi \in X_{N}(\Omega)$ such that

$$
b(\psi, \varphi)=\int_{\Omega} \tilde{f} \cdot \operatorname{curl} \varphi \quad \forall \varphi \in X_{N}(\Omega) .
$$

The bilinear form $b$ is coercive on $X_{N}(\Omega)$ by the Friedrichs coercivity estimate (3.3). This implies the solvability of problem (4.14) by some $\psi \in X_{N}(\Omega)$. We note that the solution $\psi \in X_{N}(\Omega)$ satisfies the estimate (4.10).

Step 3. The divergence of $\psi$. We claim that $\psi$ satisfies $\nabla \cdot \psi=0$.

To verify this claim, we solve, for arbitrary $\eta \in L^{2}(\Omega, \mathbb{R})$, the Dirichlet problem $\Delta \Phi=\eta$ with $\Phi \in H_{0}^{1}(\Omega)$. We want to use $\varphi:=\nabla \Phi$ as a test-function in (4.14). The construction and the regularity $\Phi \in H^{1}(\Omega)$ imply $\varphi \in X(\Omega)$ (the distributional curl vanishes, since $\varphi$ is a gradient, and the distributional divergence is $\eta$ ). Concerning the boundary condition we calculate, for test functions $\xi \in H^{2}(\Omega)$,

$$
\int_{\Omega} \operatorname{curl} \varphi \cdot \xi=\int_{\Omega} 0 \cdot \xi=0
$$

and, exploiting that $\Phi$ has vanishing boundary values,

$$
\int_{\Omega} \varphi \cdot \operatorname{curl} \xi=\int_{\Omega} \nabla \Phi \cdot \operatorname{curl} \xi=\int_{\Omega} \Phi \nabla \cdot \operatorname{curl} \xi=0 .
$$

By density, the equality of the two expressions remains valid for all test-functions $\xi \in H^{1}(\Omega)$. By definition of $X_{N}(\Omega)$, this provides $\varphi=\nabla \Phi \in X_{N}(\Omega)$. From now on, we may therefore use $\varphi$ as a test function in (4.14).

Relation (4.14) allows to calculate

$$
\begin{aligned}
0 & =\int_{\Omega} \tilde{f} \cdot \operatorname{curl} \varphi=b(\psi, \varphi) \\
& =\int_{\Omega}\{(\nabla \cdot \psi)(\nabla \cdot \varphi)+(\operatorname{curl} \psi) \cdot(\operatorname{curl} \varphi)\}=\int_{\Omega}(\nabla \cdot \psi) \eta .
\end{aligned}
$$

Since $\eta$ was arbitrary, we obtain $\nabla \cdot \psi=0$.

Step 4. Properties of the remainder. We introduce the remainder $R:=$ $\tilde{f}-\operatorname{curl} \psi$ and claim that $R$ vanishes.

We start with the observation that the property $\nabla \cdot \psi=0$ simplifies relation (4.14), which now reads

$$
\int_{\Omega} R \cdot \operatorname{curl} \varphi=\int_{\Omega}(\tilde{f}-\operatorname{curl} \psi) \cdot \operatorname{curl} \varphi=0 \quad \forall \varphi \in X_{N}(\Omega) .
$$

This shows curl $R=0$ in the sense of distributions.

Furthermore, $R$ is a solennoidal field: The divergence of $\tilde{f}$ vanishes by the construction in Step 1, and the divergence of curl $\psi$ also vanishes. 
We finally want to check the normal boundary condition for $R$. For every $\varphi \in H^{1}(\Omega)$ holds, using $\psi \in X_{N}(\Omega)$ in the last step,

$$
\int_{\Omega} R \cdot \nabla \varphi=\int_{\Omega} \tilde{f} \cdot \nabla \varphi-\int_{\Omega} \operatorname{curl} \psi \cdot \nabla \varphi \stackrel{(4.12)}{=}-\int_{\Omega} \operatorname{curl} \psi \cdot \nabla \varphi=0 .
$$

This shows $R \in X_{T}(\Omega)$.

The Friedrichs estimate (3.3) on the space $X_{T}(\Omega)$ allows to conclude from curl $R=0$ and $\operatorname{div} R=0$ the equality $R=0$. This shows the decomposition result $f=\nabla \phi+\operatorname{curl} \psi$.

Proof of Item (2). The proof of Item (2) follows along the same lines. In Step 1, the scalar potential $\phi$ is constructed as the solution $\phi \in H_{0}^{1}(\Omega)$ of the Dirichlet problem $\Delta \phi=\nabla \cdot f$. We consider the function $\tilde{f}=f-\nabla \phi$, which has vanishing divergence (but, in general, not vanishing normal boundary data). In Step 2 we consider once more the bilinear form

$$
b(u, v):=\int_{\Omega}\{(\nabla \cdot u)(\nabla \cdot v)+(\operatorname{curl} u) \cdot(\operatorname{curl} v)\},
$$

but now on the space $X_{T}(\Omega)$; the bilinear form is now $b: X_{T}(\Omega) \times X_{T}(\Omega) \rightarrow$ $\mathbb{R}$. The vector potential $\psi$ is once more constructed with the Lax-Milgram theorem; now $\psi \in X_{T}(\Omega)$ satisfies the identity of (4.14) for every test-function $\varphi \in X_{T}(\Omega)$. Step 3 can be performed as above and we obtain $\nabla \cdot \psi=0$; the test function $\varphi=\nabla \Phi$ must now be constructed by solving a Neumann problem for $\Phi$ in order to have $\varphi \in X_{T}(\Omega)$.

We provide some more details concerning Step 4: As in the proof of Item (1), we define the remainder $R:=\tilde{f}-\operatorname{curl} \psi$ and show that $R$ vanishes. By construction of $\tilde{f}$, there holds $\nabla \cdot R=0$. The fact $\nabla \cdot \psi=0$ simplifies the identity in (4.14) and we find, in analogy to relation (4.15),

$$
\int_{\Omega} R \cdot \operatorname{curl} \varphi=0 \quad \forall \varphi \in X_{T}(\Omega) .
$$

This equality shows curl $R=0$ in the sense of distributions.

Because of curl $R=0$, the equality of (4.17) is also satisfied for every function $\varphi \in X_{N}(\Omega)$. Indeed, the formal calculation for this fact is

$$
\int_{\Omega} R \cdot \operatorname{curl} \varphi=\int_{\Omega} \operatorname{curl} R \cdot \varphi=0 .
$$

The integration by parts is justified by definition of $X_{N}(\Omega)$. A rigorous proof is obtained by first regularizing $R$ and then considering the limit.

An arbitrary function $\varphi \in H^{1}(\Omega)$ can be written as the sum $\varphi=\varphi_{N}+\varphi_{T}$ with $\varphi_{N} \in X_{N}(\Omega)$ and $\varphi_{T} \in X_{T}(\Omega)$ (the proof of this fact can easily been performed using charts under the regularity assumption $\left.\partial \Omega \in \mathcal{C}^{1,1}\right)$. By linearity of the expression in $\varphi$ we obtain that the equality of (4.17) is satisfied for every function $\varphi \in H^{1}(\Omega)$. Since curl $R$ vanishes, this shows $R \in X_{N}(\Omega)$. The 
Friedrichs estimate (3.3) on the space $X_{N}(\Omega)$ allows to conclude $R=0$ and hence the decomposition result.

\section{Construction of vector potentials}

We next present a consequence on the existence of vector potentials: Given $f$ with $\nabla \cdot f=0$, we look for a vector potential $\psi$ such that curl $\psi=f$.

Classically, the construction of $\psi$ is performed with Fourier transformation methods, see [GR79]. In this approach, little regularity on $\partial \Omega$ is needed (Lipschitz is sufficient). On the other hand, without further arguments, one cannot prescribe boundary conditions for the potential $\psi$; see also [JKO94], Lemma 4.4. The results of [GR79] are stated and proved in [ABDG98].

The latter reference includes many extensions. In particular, very general domains can be considered. The notion of pseudo-Lipschitz domains is introduced and the existence of vector potentials is shown on pseudo-Lipschitz domains (domains with cuts that are not Lipschitz domains can still be pseudo-Lipschitz domains). The results of [ABDG98] include boundary conditions, see Theorems 3.12 and 3.17 of that reference.

The following result makes a strong statement on the existence of vector potentials. We note that we have to assume a high regularity of the domain. Our emphasis is on the fact that, essentially, the result can be obtained from Friedrichs inequality (3.3). We use the boundary regularity only in Item (2), compare Remark 4.3.

Corollary 5.1. Let $\Omega \subset \mathbb{R}^{3}$ be a simply connected bounded Lipschitz domain with $\partial \Omega \in \mathcal{C}^{1,1}$. Then there exists a constant $C_{V}>0$ such that, for every $f \in L^{2}\left(\Omega, \mathbb{R}^{3}\right)$, we have:

(1) $f$ with boundary condition. If $f$ has vanishing divergence and vanishing normal boundary data, i.e. $f \in W_{0}$ of (4.2), then there exists a vector potential $\psi \in X_{N}(\Omega)$ with

$$
f=\operatorname{curl} \psi, \quad\|\psi\|_{H^{1}(\Omega)} \leq C_{V}\|f\|_{L^{2}(\Omega)} .
$$

(2) $f$ without boundary condition. If $f$ has vanishing divergence, i.e. $f \in W$ of (4.4), then there exists a vector potential $\psi \in X_{T}(\Omega)$ with

$$
f=\operatorname{curl} \psi, \quad\|\psi\|_{H^{1}(\Omega)} \leq C_{V}\|f\|_{L^{2}(\Omega)} .
$$

Proof. Item (1). We use the Helmholtz decomposition according to (4.8), $f=$ $\nabla \phi+\operatorname{curl} \psi$ with $\phi \in H^{1}(\Omega, \mathbb{R})$ and $\psi \in X_{N}(\Omega)$. Upon multiplication with the gradient $\nabla \varphi$ of a test function $\varphi \in H^{1}(\Omega)$, we obtain

$$
0 \stackrel{f \in W_{0}}{=} \int_{\Omega} f \cdot \nabla \varphi=\int_{\Omega}(\nabla \phi+\operatorname{curl} \psi) \cdot \nabla \varphi \stackrel{\psi \in X_{N}}{=} \int_{\Omega} \nabla \phi \cdot \nabla \varphi .
$$

This shows that $\phi$ solves the homogeneous Neumann problem $\Delta \phi=0$ and is therefore constant. This shows $\nabla \phi=0$ and hence $f=\operatorname{curl} \psi$. 
Item (2). The proof is analogous to that of Item (1). We now use the Helmholtz decomposition according to $(4.9), f=\nabla \phi+\operatorname{curl} \psi$ with $\phi \in H_{0}^{1}(\Omega, \mathbb{R})$ and $\psi \in X_{T}(\Omega)$. Testing $f=\nabla \phi+\operatorname{curl} \psi$ with the gradient $\nabla \varphi$ of a test function $\varphi \in H_{0}^{1}(\Omega)$, we obtain that $\phi$ solves the homogeneous Dirichlet problem $\Delta \phi=0$. This shows $\phi=0$ and hence $\nabla \phi=0$. We obtain $f=\operatorname{curl} \psi$ and have therefore found the vector potential.

\section{Global Div-Curl Lemma}

One of our motivations to study the above classical decomposition results is the div-curl lemma. Most often, this lemma is formulated in a local version, with the claim that the product $f_{k} \cdot p_{k}$ of two weakly convergent sequences converges in the sense of distributions. We are interested here in global results, i.e. in results that provide the convergence of the integrals $\int_{\Omega} f_{k} \cdot p_{k}$.

Lemma 6.1 (Global div-curl lemma). Let $\Omega \subset \mathbb{R}^{3}$ be a simply connected bounded Lipschitz domain with $\partial \Omega \in \mathcal{C}^{1,1}$. Let $f_{k} \rightarrow f$ in $L^{2}\left(\Omega, \mathbb{R}^{3}\right)$ and $p_{k} \rightarrow p$ in $L^{2}\left(\Omega, \mathbb{R}^{3}\right)$ be two weakly convergent sequences. We assume that the distributional derivatives satisfy, for some $C>0$,

$$
\left\|\nabla \cdot f_{k}\right\|_{L^{2}(\Omega)} \leq C, \quad\left\|\operatorname{curl} p_{k}\right\|_{L^{2}(\Omega)} \leq C,
$$

for every $k \in \mathbb{N}$. Let furthermore be one of the two boundary conditions (i) or (ii) be satisfied for every $k \in \mathbb{N}$ :

(i) $\left.f_{k} \cdot \nu\right|_{\partial \Omega}=0$

(ii) $p_{k} \times\left.\nu\right|_{\partial \Omega}=0$

Then there holds, as $k \rightarrow \infty$,

$$
\int_{\Omega} f_{k} \cdot p_{k} \rightarrow \int_{\Omega} f \cdot p
$$

Let us include two remarks concerning the proof of the above lemma. Concerning boundary condition (i), we could rely the proof also on the convexity of the domain $\Omega$ and work without the assumption $\partial \Omega \in \mathcal{C}^{1,1}$. In the proof of boundary condition (ii), we do not exploit the boundary condition $\psi \in X_{T}(\Omega)$ on $\psi$. This means that case (ii) can be proved also with a weaker version of Theorem 4.2.

Proof. Proof for boundary condition (i). We write $p_{k}$ as $p_{k}=\nabla \phi_{k}+$ curl $\psi_{k}$ with potentials as in Theorem 4.2, Item (1), i.e. with $\psi_{k} \in X_{N}(\Omega)$ and $w_{k}:=\operatorname{curl} \psi_{k}$. We recall that $w_{k} \in W_{0}$ and hence $w_{k} \in X_{T}(\Omega)$ is satisfied, compare (4.11).

We claim that $w_{k}:=$ curl $\psi_{k}$ converges strongly in $L^{2}(\Omega)$. Indeed, we have boundedness of $w_{k}$ in $L^{2}(\Omega)$ by boundedness of $p_{k}$ in $L^{2}(\Omega)$, furthermore the obvious boundedness of $\nabla \cdot w_{k}=0$. Finally, the boundedness of curl $w_{k}=\operatorname{curl} p_{k}$ in $L^{2}(\Omega)$ holds by (6.1). The coercivity inequality (3.1) in $X_{T}(\Omega)$ provides boundedness of $w_{k}$ in $H^{1}(\Omega)$ and hence the compactness in $L^{2}(\Omega)$. 
With this compactness property for $w_{k}$ and the strong convergence $\phi_{k} \rightarrow \phi$ in $L^{2}(\Omega)$ (which follows from the compact Rellich embedding $H^{1}(\Omega) \subset L^{2}(\Omega)$ ) we can calculate

$$
\begin{aligned}
\int_{\Omega} f_{k} \cdot p_{k} & =\int_{\Omega} f_{k} \cdot\left(\nabla \phi_{k}+\operatorname{curl} \psi_{k}\right)=\int_{\Omega}\left(-\nabla \cdot f_{k}\right) \phi_{k}+f_{k} \cdot \operatorname{curl} \psi_{k} \\
& \rightarrow \int_{\Omega}(-\nabla \cdot f) \phi+f \cdot \operatorname{curl} \psi=\int_{\Omega} f \cdot(\nabla \phi+\operatorname{curl} \psi)=\int_{\Omega} f \cdot p .
\end{aligned}
$$

We used in the last step that the limits $\phi$ and $\psi$ are indeed the Helmholtz decomposition functions for the limit $p$. This provides the claim for boundary condition (i).

Proof for boundary condition (ii). We now decompose $f_{k}$ as $f_{k}=\nabla \phi_{k}+$ curl $\psi_{k}$ using Theorem 4.2, Item (2) (but we will not exploit $\psi_{k} \in X_{T}(\Omega)$ ). The a priori bound $\left\|\psi_{k}\right\|_{H^{1}(\Omega)} \leq C_{0}$ from (4.10) allows to select a subsequence $k \rightarrow \infty$ with the strong convergence $\psi_{k} \rightarrow \psi$ in $L^{2}(\Omega)$.

The functions $\phi_{k} \in H_{0}^{1}(\Omega)$ solve a Dirichlet problem: For every $\varphi \in H_{0}^{1}(\Omega)$ there holds

$$
\int_{\Omega} \nabla \phi_{k} \cdot \nabla \varphi=\int_{\Omega}\left(f_{k}-\operatorname{curl} \psi_{k}\right) \cdot \nabla \varphi=-\int_{\Omega} \nabla \cdot f_{k} \varphi .
$$

This is the weak form of the Dirichlet problem $\Delta \phi_{k}=\nabla \cdot f_{k}$. Since the solution map $H^{-1}(\Omega) \rightarrow H_{0}^{1}(\Omega)$ of this Dirichlet problem is linear and continous, the strong convergence of $\nabla \cdot f_{k}$ in $H^{-1}(\Omega)$ implies the strong convergence $\nabla \phi_{k} \rightarrow$ $\nabla \phi$ in $L^{2}(\Omega)$.

After this preparation we can calculate, using boundary condition (ii) for $p_{k}$ and for $p$, in the limit $k \rightarrow \infty$,

$$
\begin{aligned}
\int_{\Omega} f_{k} \cdot p_{k} & =\int_{\Omega}\left(\nabla \phi_{k}+\operatorname{curl} \psi_{k}\right) \cdot p_{k}=\int_{\Omega} \nabla \phi_{k} \cdot p_{k}+\psi_{k} \cdot \operatorname{curl} p_{k} \\
& \rightarrow \int_{\Omega} \nabla \phi \cdot p+\psi \cdot \operatorname{curl} p=\int_{\Omega} \nabla \phi \cdot p+\operatorname{curl} \psi \cdot p=\int_{\Omega} f \cdot p .
\end{aligned}
$$

This was the claim in (6.2).

Corollary 6.2 (The usual div-curl lemma on arbitrary domains). Let $\Omega \subset \mathbb{R}^{3}$ be an open set and let $p_{k}$ and $f_{k}$ be sequences with $f_{k} \rightarrow f$ and $p_{k} \rightarrow p$ in $L^{2}\left(\Omega, \mathbb{R}^{3}\right)$. We assume the div- and curl-control of (6.1). Then $f_{k} \cdot p_{k} \rightarrow f \cdot p$ in the sense of distributions on $\Omega$.

Proof. Upon subtracting $f$ and $p$ from the sequences, we can assume $p=0$ and $f=0$. The distributional convergence is a local property, it suffices to show that, for an arbitrary open ball $B \subset \bar{B} \subset \Omega$ and an arbitrary smooth function $\varphi \in C_{c}^{\infty}(B, \mathbb{R})$ there holds

$$
\int_{B} f_{k} \cdot p_{k} \varphi \rightarrow 0
$$


The relation (6.3) is a direct consequence of the global div-curl lemma 6.1 (ii), applied to the sequences $f_{k}$ and $p_{k} \varphi$. The sequence $p_{k} \varphi$ converges weakly to 0 , has $L^{2}(B)$-bounded curl and satisfies the homogeneous tangential boundary condition. The ball $B$ has a $\mathcal{C}^{1,1}$-boundary. Lemma 6.1 provides (6.3) and thus the claim.

\section{Comments And Generalizations}

We emphasize that there is another route to prove the above div-curl result. One can start the analysis from the simple Helmholtz decomposition of Proposition 4.1. This requires no properties of $\Omega$. When needed, one can use Theorem 3.12 or 3.17 of [ABDG98] to write a solennoidal field $w$ as a curl, $w=\operatorname{curl} \psi$. This requires less regularity on $\Omega$ than our Corollary 5.1 (essentially, Lipschitz domains with cuts are allowed). We make this observation more precise with the following remark.

Remark 7.1 (Global div-curl lemma on Lipschitz domains). The statement of Lemma 6.1 remains valid on general bounded Lipschitz domains $\Omega$.

Proof. Let us start with the boundary condition (i). We proceed as in the proof of Lemma 6.1 (i) and decompose $p_{k}$. We use the simple Helmholtz decomposition of Proposition 4.1, Item (1), and write $p_{k}=\nabla \phi_{k}+w_{k}$ with $\phi_{k} \in H^{1}(\Omega)$ and $w_{k} \in W_{0}$. We use the existence result for vector potentials from Theorem 3.17 of [ABDG98]: there exists a potential $\psi_{k} \in X_{N}(\Omega)$ with $w_{k}=\operatorname{curl} \psi_{k}$. The boundary condition for $\psi_{k}$ allows to conclude strong convergence of $w_{k}$ from

$$
\begin{aligned}
\int_{\Omega}\left|w_{k}\right|^{2} & =\int_{\Omega} w_{k} \cdot \operatorname{curl} \psi_{k}=\int_{\Omega} \operatorname{curl} w_{k} \cdot \psi_{k}=\int_{\Omega} \operatorname{curl} p_{k} \cdot \psi_{k} \\
& \rightarrow \int_{\Omega} \operatorname{curl} p \cdot \psi=\int_{\Omega} w \cdot \operatorname{curl} \psi=\int_{\Omega}|w|^{2} .
\end{aligned}
$$

The proof for boundary condition (ii) follows closely the one of Lemma 6.1 (ii) and uses no boundary conditions for the potentials $\psi_{k}$. We decompose $f_{k}=\nabla \phi_{k}+w_{k}$ with $\phi_{k} \in H_{0}^{1}(\Omega)$ and exploit the strong convergence of $\nabla \phi_{k}$. The functions $w_{k} \in W$ are written as $w_{k}=\operatorname{curl} \psi_{k}$. In the calculation of the integral, we can integrate by parts in the term $\left(\operatorname{curl} \psi_{k}\right) \cdot p_{k}$, due to the boundary condition for $p_{k}$.

We use this opportunity to include a simple remark: In the case of higher integrability properties of the functions, general domains and the case without boundary conditions can be treated.

Remark 7.2 (Global div-curl lemma on arbitrary domains). The statement of Lemma 6.1 without boundary conditions on either $p_{k}$ or $f_{k}$ remains valid on general bounded domains $\Omega$ if the sequence $f_{k}$ (or the sequence $p_{k}$ ) is bounded in $L^{q}(\Omega)$ for some $q>2$. 
Proof. The sequence $f_{k} \cdot p_{k}$ is bounded in the reflexive space $L^{1+\delta}(\Omega)$ for some $\delta>0$. It therefore converges weakly in $L^{1+\delta}(\Omega)$ to its distributional limit, which is $f \cdot p$ by Corollary 6.2. The weak convergence implies the convergence of integrals (6.2). This proves the claim.

\section{REFERENCES}

[ABDG98] C. Amrouche, C. Bernardi, M. Dauge, and V. Girault. Vector potentials in threedimensional non-smooth domains. Math. Methods Appl. Sci., 21(9):823-864, 1998.

[Cos91] M. Costabel. A coercive bilinear form for Maxwell's equations. J. Math. Anal. Appl., 157(2):527-541, 1991.

[GR79] V. Girault and P.-A. Raviart. Finite element approximation of the Navier-Stokes equations, volume 749 of Lecture Notes in Mathematics. Springer-Verlag, BerlinNew York, 1979.

[HS14] M. Heida and B. Schweizer. Stochastic homogenization of a plasticity system. Technical Report 2014-08, TU Dortmund, 2014.

[HS15] M. Heida and B. Schweizer. Non-periodic homogenization of infinitesimal strain plasticity equations. Z. Angew. Math. Mech., (DOI 10.1002/zamm.201400112):119, 2015.

[JKO94] V. Jikov, S. Kozlov, and O. Oleinik. Homogenization of Differential Operators and Integral Functionals. Springer, 1994.

[KN84] M. Kř́žzek and P. Neittaanmäki. On the validity of Friedrichs' inequalities. Math. Scand., 54(1):17-26, 1984.

[Lei68] R. Leis. Zur Theorie elektromagnetischer Schwingungen in anisotropen inhomogenen Medien. Math. Z., 106:213-224, 1968.

[Sar82] J. Saranen. On an inequality of Friedrichs. Math. Scand., 51(2):310-322 (1983), 1982.

[SV11] B. Schweizer and M. Veneroni. The needle problem approach to non-periodic homogenization. Netw. Heterog. Media, 6(4):755-781, 2011.

[vW92] W. von Wahl. Estimating $\nabla u$ by $\operatorname{div} u$ and curl $u$. Math. Methods Appl. Sci., 15(2):123-143, 1992. 


\section{Preprints ab 2013/07}

2016-03

2016-02

2016-01

2015-13

2015-12

2015-11

2015-10

2015-09

2015-08

2015-07

2015-06

2015-05

2015-04

2015-03

2015-02

2015-01

2014-10

\section{Ben Schweizer}

On Friedrichs inequality, Helmholtz decomposition, vector potentials, and the div-curl lemma

\section{Michael Voit}

Generalized commutative association schemes, hypergroups, and positive product formulas

\section{Agnes Lamacz and Ben Schweizer}

Effective acoustic properties of a meta-material consisting of small Helmholtz resonators

\section{Christian Eggert, Ralf Gäer, Frank Klinker}

The general treatment of non-symmetric, non-balanced star circuits: On the geometrization of problems in electrical metrology

Daniel Kobe and Jeannette H.C. Woerner

Oscillating Ornstein-Uhlenbeck processes and modelling electricity prices

\section{Sven Glaser}

A distributional limit theorem for the realized power variation of linear fractional stable motions

\section{Herold Dehling, Brice Franke and Jeannette H.C. Woerner}

Estimating drift parameters in a fractional Ornstein Uhlenbeck process with periodic mean

\section{Harald Garcke, Johannes Kampmann, Andreas Rätz and Matthias Röger}

A coupled surface-Cahn-Hilliard bulk-diffusion system modeling lipid raft formation in cell membrans

\section{Agnes Lamacz and Ben Schweizer}

Outgoing wave conditions in photonic crystals and transmission properties at interfaces

Manh Hong Duong, Agnes Lamacz, Mark A. Peletier and Upanshu Sharma

Variational approach to coarse-graining of generalized gradient flows

\section{Agnes Lamacz and Ben Schweizer}

A negative index meta-material for Maxwell's equations

Michael Voit

Dispersion and limit theorems for random walks associated with hypergeometric functions of type $B C$

\section{Andreas Rätz}

Diffuse-interface approximations of osmosis free boundary problems

Margit Rösler and Michael Voit

A multivariate version of the disk convolution

\section{Christina Dörlemann, Martin Heida, Ben Schweizer}

Transmission conditions for the Helmholtz-equation in perforated domains

\section{Frank Klinker}

Program of the International Conference

Geometric and Algebraic Methods in Mathematical Physics

March 16-19, 2015, Dortmund

\section{Frank Klinker}

An explicit description of SL $(2, \mathbb{C})$ in terms of $\mathrm{SO}^{+}(3,1)$ and vice versa 
Integral representation and sharp asymptotic results for some Heckman-Opdam hypergeometric functions of type BC

2014-08 Martin Heida and Ben Schweizer

Stochastic homogenization of plasticity equations

2014-07 Margit Rösler and Michael Voit

A central limit theorem for random walks on the dual of a compact Grassmannian

2014-06 Frank Klinker

Eleven-dimensional symmetric supergravity backgrounds, their geometric superalgebras, and a common reduction

Bifurcation of nonlinear Bloch waves from the spectrum in the Gross-Pitaevskii equation

2014-04 Frank Klinker

A family of non-restricted $D=11$ geometric supersymmetries

2014-03 Martin Heida and Ben Schweizer

Non-periodic homogenization of infinitesimal strain plasticity equations

2014-02 Ben Schweizer

The low frequency spectrum of small Helmholtz resonators

2014-01 Tomáš Dohnal, Agnes Lamacz, Ben Schweizer

Dispersive homogenized models and coefficient formulas for waves in general periodic media

2013-16 Karl Friedrich Siburg

Almost opposite regression dependence in bivariate distributions

2013-15 Christian Palmes and Jeannette H. C. Woerner

The Gumbel test and jumps in the volatility process

2013-14 Karl Friedrich Siburg, Katharina Stehling, Pavel A. Stoimenov, Jeannette H. C. Wörner

An order for asymmetry in copulas, and implications for risk management

2013-13 Michael Voit

Product formulas for a two-parameter family of Heckman-Opdam hypergeometric functions of type BC

2013-12 Ben Schweizer and Marco Veneroni

Homogenization of plasticity equations with two-scale convergence methods

2013-11 Sven Glaser

A law of large numbers for the power variation of fractional Lévy processes

2013-10 Christian Palmes and Jeannette H. C. Woerner

The Gumbel test for jumps in stochastic volatility models

2013-09 Agnes Lamacz, Stefan Neukamm and Felix Otto

Moment bounds for the corrector in stochastic homogenization of a percolation model

2013-08 Frank Klinker

Connections on Cahen-Wallach spaces

2013-07 Andreas Rätz and Matthias Röger

Symmetry breaking in a bulk-surface reaction-diffusion model for signaling networks 
